\title{
Array Regrouping and Structure Splitting Using Whole-Program Reference Affinity
}

\author{
Yutao Zhong, Maksim Orlovich, Xipeng Shen, and Chen Ding \\ Computer Science Department \\ University of Rochester \\ \{ytzhong, orlovich, xshen, cding\}@cs.rochester.edu
}

\begin{abstract}
While the memory of most machines is organized as a hierarchy, program data are laid out in a uniform address space. This paper defines a model of reference affinity, which measures how close a group of data are accessed together in a reference trace. It proves that the model gives a hierarchical partition of program data. At the top is the set of all data with the weakest affinity. At the bottom is each data element with the strongest affinity. Based on the theoretical model, the paper presents $k$-distance analysis, a practical test for the hierarchical affinity of source-level data. When used for array regrouping and structure splitting, $k$-distance analysis consistently outperforms data organizations given by the programmer, compiler analysis, frequency profiling, statistical clustering, and all other methods we have tried.
\end{abstract}

\section{INTRODUCTION}

All current PCs and workstations use cache blocks of at least 64 bytes, making the utilization an important problem. If only one word is useful in each cache block, a cache miss will not serve as a prefetch for other useful data. Furthermore, the program would waste up to $93 \%$ of memory transfer bandwidth and $93 \%$ of cache space, causing even more memory access.

To improve cache utilization we need to group related data into the same cache block. The question is how to define the relation. We believe that it should meet three requirements. First, it should be solely based on how data are accessed. For example in an access sequence "abab..ab", $a$ and $b$ are related and should be put in the same cache block, regardless how they are allocated and whether they are linked by pointers. Second, the relation must give a unique partition of data. Consider for example the access sequence " $a b a b . . a b . . . b c b c . . b c$ ". Since data $a$ and $c$ are not related, $b$ cannot relate to both of them because it cannot stay in two locations in memory. Finally, the relation should be a scale. The block of different memory levels is increasingly large, from cache line to memory page. After grouping "most related" data into the smallest block, we need to group "next related" data into larger blocks. In summary, the relation should give a unique and hierarchical organization of all program data.

In this paper, we define such a relation we call reference affinity, which measures how close a group of data are accessed together in an execution. Unlike most other program analysis, we measure the "togetherness" using the LRU stack distance, defined as the amount of data accessed between two memory references in an execution trace [15]. Like locality, stack distance is bounded, even for longrunning programs. The long distance often reveals long-range data access patterns that may otherwise hide behind complex control flows, indirect data access, or variations in coding and data allocation. We prove that the new definition gives a unique partition of program data for each distance $k$. When we decrease the value of $k$, reference affinity gives a hierarchical decomposition and finds data sub-groups with closer affinity, much in the same way we sharpen the focus by reducing the radius of a circle.

Two earlier studies defined a reuse signature as a histogram of the reuse distance of all program data access $[9,20]$. They showed that the reuse signature has a consistent pattern across all data inputs even for complex programs or regular programs after complex compiler optimizations. This suggests that we can analyze the reference affinity of the whole program by looking at its reuse signatures from training runs.

We present $k$-distance analysis, which simplifies the requirements of reference affinity into a set of necessary conditions about reuse signatures. The simplified conditions can then be checked efficiently for large, complex programs. The parameter $k$ has an intuitive meaning - elements in the same group are almost always used within a distance of $k$ data elements. By varying the value of $k$, the analysis gives the hierarchical affinity relation among data arrays and structure fields. By picking different $k$, it gives appropriate data layout for different levels of memory hierarchy, from a single cache block to physical memory. The analysis handles sequential programs with arbitrarily complex control flow, indirect data access, and dynamic memory allocation. The analysis uses multiple training runs to take into account the variation caused by program inputs.

Although reference affinity and $k$-distance analysis have strong properties, they are not optimal. We will formulate the problems in terms of partial and dynamic reference affinity and discuss their complexity. Our analysis checks the necessary rather than the sufficient conditions of reference affinity, so it may include data with false affinity. We will show that the probability of error is small and can be further reduced by strengthening the conditions.

The rest of the paper is organized as follows. Section 2 defines the formal model of reference affinity and proves its properties. Section 3 presents $k$-distance analysis and a comparison with a number of other methods. Section 4 describes the compiler support for array regrouping and structure splitting. The last three sections present the experimental evaluation, related work, and a summary.

\section{MODEL OF REFERENCE AFFINITY}

This section first defines three preliminary concepts and gives two examples of our reference affinity model. Then it presents the formal definition and proves the properties of our model including 
consistent affinity and hierarchical organization.

An address trace or reference string is a sequence of accesses to a set of data elements. If we assign a logical time to each access, the address trace is a vector indexed by the logical time. We use letters such as $x, y, z$ to represent data elements, subscripted symbols such as $a_{x}, a_{x}^{\prime}$ to represent accesses to a particular data element $x$, and the array index $T\left[a_{x}\right]$ to represent the logical time of the access $a_{x}$ on trace $T$.

The $L R U$ stack distance between two accesses, $a_{x}$ and $a_{y}\left(T\left[a_{x}\right]\right.$ $\left.<T\left[a_{y}\right]\right)$, in a trace $T$ is the number of distinct data elements accessed in times $T\left[a_{x}\right], T\left[a_{x}\right]+1, \ldots, T\left[a_{y}\right]-1$. We write it as $\operatorname{dis}\left(a_{x}, a_{y}\right)$. If $T\left[a_{x}\right]>T\left[a_{y}\right]$, we let $\operatorname{dis}\left(a_{x}, a_{y}\right)=\operatorname{dis}\left(a_{y}, a_{x}\right)$. If $T\left[a_{x}\right]=T\left[a_{y}\right]$, dis $\left(a_{x}, a_{y}\right)=0$. The distance is the volume of data accessed between two points of a trace, so we also call it volume distance. In contrast, time distance is the difference between the logical time of two accesses. For example, the volume distance between the accesses to $a$ and $c$ in trace $a b b b c$ is 2, while the time distance is 4 . The volume distance is Euclidean. Given any three accesses in the time order, $a_{x}, a_{y}$, and $a_{z}$, we have $\operatorname{dis}\left(a_{x}, a_{z}\right) \leq \operatorname{dis}\left(a_{x}, a_{y}\right)+\operatorname{dis}\left(a_{y}, a_{z}\right)$, because the cardinality of the union of two sets is no greater than the sum of the cardinality of each set.

Based on volume distance, we define a linked path on a trace. It is parameterized by a distance bound $k$. There is a linked path from $a_{x}$ to $a_{y}(x \neq y)$ if and only if there exist $t$ accesses, $a_{x_{1}}$, $a_{x_{2}}, \ldots, a_{x_{t}}$, such that (1) $\operatorname{dis}\left(a_{x}, a_{x_{1}}\right) \leq k \wedge \operatorname{dis}\left(a_{x_{1}}, a_{x_{2}}\right) \leq$ $k \wedge \ldots \wedge \operatorname{dis}\left(a_{x_{t}}, a_{y}\right) \leq k$ and (2) $x_{1}, x_{2}, \ldots, x_{t}, x$ and $y$ are different data elements. In other words, a linked path is a sequence of accesses to different data elements, and each link (between two consecutive members of the sequence) has a volume distance no greater than $k$. We call $k$ the link length. We will later restrict $x_{1}, x_{2}, \ldots, x_{t}$ to be members of some set $S$. If so, we say that there is a linked path from $a_{x}$ to $a_{y}$ with link length $k$ for set $S$.

We now explain reference affinity with two example address traces in Figure 1. The "..." represents accesses to data other than $w, x, y$, and $z$. In the first example, accesses to $x, y$, and $z$ are in three periods. The three elements belong to the same affinity group because they are always accessed together. The consistency is important for data placement. For example, $x$ and $w$ are not always used together, then putting them into the same cache block would waste cache space when only one of the two is accessed. The example shows that finding this consistency is not trivial. The accesses to the three data elements appear in different orders, with different frequency, and mixed with accesses to other data. However, one property holds in all three time ranges - the accesses to the three elements are connected by a linked path with link length 2 .

xyz .. xwzzy .. yzuvvrvvx ...

(1) The affinity group $\{x, y, z\}$ with link length $k=2$

wxwxuyzyz ... zyzyvwxwx ...

(2) The affinity group $\{\mathrm{w}, \mathrm{x}, \mathrm{y}, \mathrm{z}\}$ at $k=2$ becomes two groups $\{\mathrm{w}, \mathrm{x}\}$ and $\{\mathrm{y}, \mathrm{z}\}$ at $k=1$

\section{Figure 1: Examples of reference affinity model and its proper-} ties

As we will prove later, affinity groups are parameterized by the link length $k$, and they form a partition of program data for each $k$. The second example in Figure 1 shows that this group partition has a hierarchical structure. The affinity group with the link length of 2 is $\{w, x, y, z\}$. If we reduce the link length to 1 , the two new groups will be $\{w, x\}$ and $\{y, z\}$. The structure is hierarchical with respect to the link length: groups at a smaller link length are subsets of groups at a greater link length. The hierarchical structure is useful in data placement because it may find different-sized affinity groups that match the capacity of the multi-level cache hierarchy.

We now present the formal definition of reference affinity.

Definition 1. Strict Reference Affinity. Given an address trace, a set $G$ of data elements is a strict affinity group (i.e. they have reference affinity) with link length $k$ if and only if

1. for any $x \in G$, all its accesses $a_{x}$ must have a linked path from $a_{x}$ to some $a_{y}$ for each other member $y \in G$, that is, there exist different elements $x_{1}, x_{2}, \ldots, x_{t} \in G$ such that $\operatorname{dis}\left(a_{x}, a_{x_{1}}\right) \leq k \wedge \operatorname{dis}\left(a_{x_{1}}, a_{x_{2}}\right) \leq k \wedge \ldots \wedge \operatorname{dis}\left(a_{x_{t}}, a_{y}\right) \leq$ $k$

2. adding any other element to $G$ will make Condition (1) impossible to hold

The following theorem proves that strict affinity groups are consistent because they form a partition of program data. In other words, each data element belongs to one and only one affinity group.

THEOREM 1. Given an address trace and a link length $k$, the affinity groups defined by Definition 1 form a unique partition of program data.

Proof. We show that any element $x$ of program data belongs to one and only one affinity group at a link length $k$. For the "one" part, observe that Condition (1) in Definition 1 holds trivially when $x$ is the only member of a group. Therefore any element must belong to some affinity group.

We prove the "only-one" part by contradiction. Suppose $x$ belongs to $G_{1}$ and $G_{2}\left(G_{1} \neq G_{2}\right)$. Then we can show that $G_{3}=$ $G_{1} \cup G_{2}$ satisfies Condition (1). For any two elements $y, z \in G_{3}$, if both belong to $G_{1}$ and $G_{2}$, then Condition (1) holds.

Without loss of generality, assume $y \in G_{1} \wedge y \notin G_{2}$ and $z \in$ $G_{2} \wedge z \notin G_{1}$. Because $y, x \in G_{1}$, any $a_{y}$, must have a linked path to an $a_{x}$, that is, there exist $y_{1}, \ldots, y_{t} \in G_{1}$ and an access $a_{x}$ such that $\operatorname{dis}\left(a_{y}, a_{y_{1}}\right) \leq k \wedge \ldots \wedge \operatorname{dis}\left(a_{y_{t}}, a_{x}\right) \leq k$. Similarly, there is a linked path for this $a_{x}$ to an $a_{z}$ because $x, z \in G_{2}$, that is, there exist $z_{1}, \ldots, z_{m} \in G_{2}$ and an access $a_{z}$ such that $\operatorname{dis}\left(a_{x}, a_{z_{1}}\right) \leq$ $k \wedge \ldots \wedge \operatorname{dis}\left(a_{z_{m}}, a_{z}\right) \leq k$.

If $y_{1}, \ldots, y_{t} \notin\left\{z_{1}, \ldots, z_{m}\right\}$, then there is a linked path from $a_{y}$ to some $a_{z}$. Suppose $y_{1}, \ldots, y_{i-1} \notin\left\{z_{1}, \ldots, z_{m}\right\}$ but $y_{i}=$ $z_{p}$. Then we have a linked path from $a_{y}$ to $a_{y_{i}}$. Since $y_{i}=$ $z_{p} \in G_{2}$, there is a linked path from $y_{i}$ to $z$, that is, there exist $z_{1}^{\prime}, z_{2}^{\prime}, \ldots, z_{n}^{\prime} \in G_{2}$ such that $\operatorname{dis}\left(a_{y}, a_{y_{1}}\right) \leq k \wedge \ldots \wedge \operatorname{dis}\left(a_{y_{i_{1}}}, a_{y_{i}}\right)$ $\leq k \wedge \operatorname{dis}\left(a_{y_{i}}, a_{z_{1}^{\prime}}\right) \wedge \ldots \wedge \operatorname{dis}\left(a_{z_{n}}^{\prime}, a_{z}\right) \leq k$. Now $y_{i}$ belongs to $G_{1} \cap G_{2}$, just like $x$. We have come back to the same situation except the linked path from $a_{y}$ to $a_{y_{i}}$ is shorter than the path from $a_{y}$ to $a_{x}$. We repeat this process. If $y_{1}, \ldots, y_{i-1} \notin\left\{z_{1}^{\prime}, \ldots, z_{n}^{\prime}\right\}$, then we have a linked path from $a_{y}$ to $a_{z}$. Otherwise, there must be $y_{j} \in\left\{z_{1}^{\prime}, \ldots, z_{n}^{\prime}\right\}$ for some $j<i$. The process cannot repeat forever because each step shortens the path from $y$ to the access chosen next by this process. It must terminate in a finite number of steps. We then have a linked path from $a_{y}$ to $a_{z}$ in $G_{3}$. Therefore, Condition (1) always holds for $G_{3}$. Since $G_{1}, G_{2} \subset G_{3}$, they are not the largest sets that satisfy Condition (1). Therefore, Condition (2) 
does not hold for $G_{1}$ or $G_{2}$. A contradiction. Therefore, $x$ belongs to only one affinity group, and affinity groups form a partition.

For a fixed link length, the partition is unique. Suppose more than one types of partition can result from Definition 1, then some $x$ belongs to $G_{1}$ in one partition and $G_{2}$ in another partition. As we have just seen, this is not possible because $G_{3}=G_{1} \cup G_{2}$ satisfies Condition (1) and therefore neither $G_{1}$ nor $G_{2}$ is an affinity group.

As we just proved, reference affinity is consistent because all members will always be accessed together (i.e. linked by some linked path with the link length $k$ ). The consistency means that packing data in an affinity group will always improve cache utilization. In addition, the group partition is unique because each data element belongs to one and only one group for a fixed $k$. The uniqueness removes any possible conflict, which would happen if a data element could appear in multiple affinity groups.

Next we prove that strict reference affinity has a hierarchical structure - an affinity group with a shorter link length is a subset of an affinity group with a greater link length.

THEOREM 2. Given an address trace and two distances $k$ and $k^{\prime}\left(k<k^{\prime}\right)$, the affinity groups at $k$ form a finer partition of affinity groups at $k^{\prime}$.

Proof. We show that any affinity group at $k$ is a subset of some affinity group at $k^{\prime}$. Let $G$ be an affinity group at $k$ and $G^{\prime}$ be the affinity group at $k^{\prime}$ that overlaps with $G\left(G \cap G^{\prime} \neq \emptyset\right)$. Since any $x, y \in G$ are connected by a linked path with link length $k$, they are connected by a linked path with a larger link length $k^{\prime}$. According to the proof of Theorem 1, $G \cup G^{\prime}$ is an affinity group at $k^{\prime}$. $G$ must be a subset of $G^{\prime}$; otherwise $G^{\prime}$ is not an affinity group because it can be expanded while still guaranteeing Condition (1).

Finally, we show that elements of the same affinity group are always accessed together. When one element is accessed, all other elements will be accessed within a bounded volume distance.

THEOREM 3. Given an address trace with an affinity group $G$ at link length $k$, any time an element $x$ of $G$ is accessed at $a_{x}$, there exists a trace segment that includes $a_{x}$, in which all other members of $G$ are accessed at least once. The volume distance between the two ends of the segment is no greater than $2 k|G|+1$, where $|G|$ is the number of elements in the affinity group.

Proof. According to Definition 1 , for any $y$ in $G$, there is a linked path from $a_{x}$ to some $a_{y}$. Sort these accesses in time order. Let $a_{w}$ be the earliest and $a_{v}$ be the latest in the trace. There is a linked path from $a_{w}$ to $a_{x}$. Let the sequence be $a_{x_{1}}, a_{x_{2}}, \ldots, a_{x_{t}}$. The volume distance from $a_{w}$ to $a_{x}$ is $\operatorname{dis}\left(a_{w}, a_{x}\right)$. It is no greater than $\operatorname{dis}\left(a_{w}, a_{x_{1}}\right)+\operatorname{dis}\left(a_{x_{1}}, a_{x_{2}}\right)+\ldots+\operatorname{dis}\left(a_{x_{t}}, a_{x}\right)$, which is $(t+1) k \leq|G| k$. The bound of the volume distance from $a_{x}$ to $a_{v}$ is the same. Considering that $a_{v}$ needs to be included in the segment, the total volume distance is at most $2 k|G|+1$.

The strict affinity requires that members of an affinity group are always accessed together. Ding and Kennedy showed that it gives the best data layout when no side effect (i.e. increased cache misses) is allowed [6]. On most machines, it is still profitable to group data that are almost always accessed together because the side effect would not out-weight the benefit. For programs with different behavior phases, it may be profitable to exploit reference affinity in each phase and change data layout between phases. We call these extensions partial reference affinity and dynamic reference affinity.
Ding and Kennedy showed that the optimal data layout in these two cases is machine dependent and finding the optimal layout is an NP-hard problem [6]. Next we present a method that measures the "almost strict" reference affinity in complex programs. The link length $k$ will play a critical role as it did in this section.

\section{WHOLE-PROGRAM AFFINITY}

We now study the reference affinity among source-level data. We specifically target data arrays and instances of structure fields because they account for major portions of global and heap data in most programs. Since an array or a field represents a set of data, we need to extend the affinity definition. The affinity exists among data sets if the sets have the same number of elements, and one element in one set has reference affinity with one and only one unique element in every other set. In particular, reference affinity exists for two arrays, $A$ and $B$, if it exists for $A[i]$ and $B[i]$ for all $i$. The affinity exists for structure fields, $f_{1}$ and $f_{2}$, if it exists for $o . f_{1}$ and $o . f_{2}$ for all instance $o$. Next, we introduce the reuse signature, the basis for whole-program affinity analysis. We then present $k$-distance analysis and a comparison with other methods.

\subsection{Reuse signature}

The reuse signature of a set of data is the histogram of the reuse distance of their accesses. Ding and Zhong showed that the wholeprogram reuse signature exhibits a consistent pattern across data inputs in a surprisingly large number of complex programs including 13 Spec95 and Spec2K integer and floating-point benchmarks [9]. The result suggests that we can use the reuse signature from one profiling run to infer the reuse signatures in other executions. Therefore, reuse signature allows not just whole-trace analysis but also whole-program analysis of reference affinity.

We illustrate the use of reuse signature through an example program, Cheetah, a fully associative LRU cache simulator that is part of the SimpleScalar 3.0 tool set. The main data structure is a splay tree, and each tree node has a number of fields, of which we concern three in this example to save space. Based on a profile from a simple input, we draw the accesses to the three fields on time-space graphs shown in Figure 2. Each access is a point whose $x$-axis value is the logical time (in memory references) and $y$-axis the memory address. The similarity of the graphs suggests that the two fields $r t w t$ (the sub-tree weight) and lft (left-child pointer) have reference affinity because they seem to be always accessed together. The third field, $a d d r$, is accessed only occasionally together with the other two fields. A manual inspection of the splay tree algorithm confirms these conjectures. The program uses the first two fields together for tree rotation involved in every step of a tree search. The tree is indexed by time not address, so the program touches the third field only at the end of a tree search.

Figure 3 shows the reuse signature of the three fields, which is a histogram of the reuse distance of accesses with a distance no smaller than 1024 to each field. The $x$-axis is a sequence of bins representing different ranges of reuse distance. The bins may be of the same size (linear scale), exponential size (log scale as in this example), or their combination (log-linear scale). The $y$-axis gives the number of memory accesses whose reuse distance falls into each bin.

Let's compare the reuse signature of $r t w t$ and lft. The latter has more reuses at the first bin because it is repeatedly accessed during initialization. However, the additional reuses have a short distance and do not affect reference affinity. The number of reuses in the second and third bins also differ. This is because many reuses with 

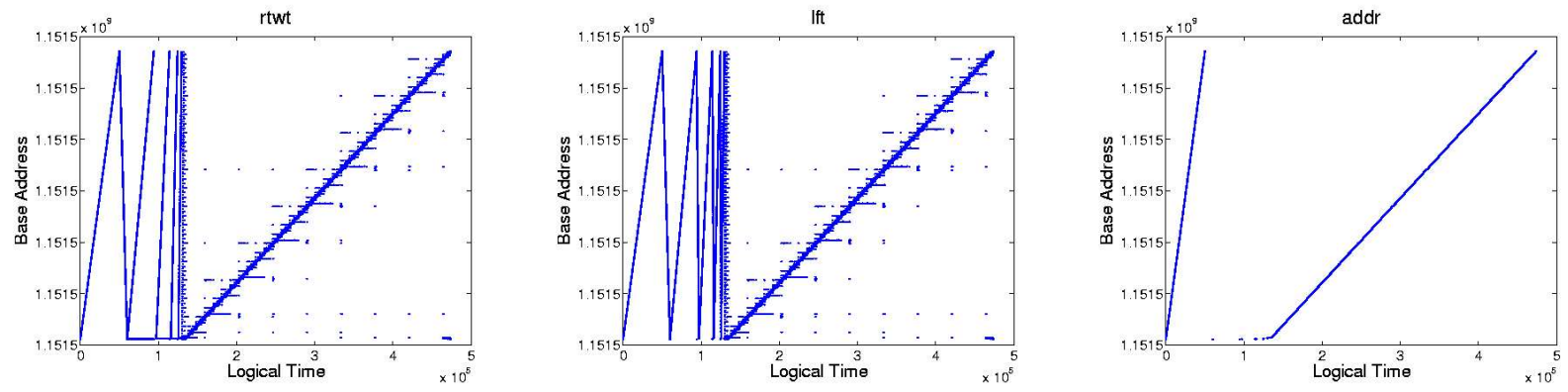

Figure 2: Time-space graphs of accesses to three tree-node fields

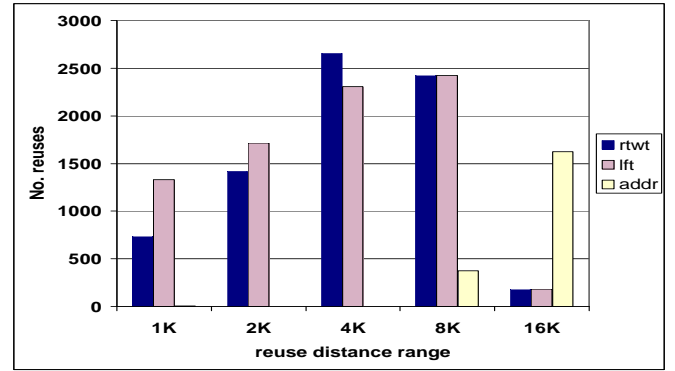

Figure 3: Reuse signatures of three tree-node fields

a distance close to $4 \mathrm{~K}$ are separated by the arbitrary bin boundary. The total number of reuses with a distance more than $2 \mathrm{~K}$ is very similar. In the last two (larger) bins, rtwt and lft have the same number of accesses, but the number is different for $a d d r$.

We use the reuse signature for affinity analysis as follows. We treat reuse signatures as vectors, remove the first few elements (reuses whose distance is shorter than 2048), and find affinity groups by comparing the reuse signatures. As shown later, we will find the correct affinity relation. A remaining question is whether other inputs to Cheetah have the same affinity. As a simulator, the access pattern in Cheetah should depend completely on the input. After checking a few other inputs, we found that the consistency remains: the time-space graph and the upper portion of the reuse signature remain identical between $r t w t$ and $l f t$ but different from $a d d r$. In fact, we can consider multiple training runs by combining their reuse signatures and consequently rule out false affinity appeared in one input but not others. By comparing reuse signatures, we find the reference affinity in Cheetah despite that the access pattern is hidden behind branches, recursive functions, and pointer indirections.

We have converted the problem from checking reference affinity in a trace, to checking patterns in time-space graph, and finally to checking the similarity of reuse signature. The compression of information is dramatic: from billions of memory accesses and timespace pairs to vectors of $\log M$ elements, where $M$ is the size of program data (the maximal reuse distance). Next we inject rigor into the use of reuse signature by formulating it in terms of the necessary conditions of reference affinity.

\subsection{K-distance analysis}

$K$-distance analysis finds reference affinity by checking a necessary (but not sufficient) condition on the data reuse signature. We first derive this condition for two data elements, then extend it to two or more data sets, and finally discuss the improvements to the condition.

As a basic case, let an affinity group have two elements, $x$ and $y$, always accessed within a link length $k$. After removing shortdistance reuses, the number of accesses to $x, N_{x}$, should be no more than the number of accesses to $y, N_{y}$. Otherwise, two accesses of $x$ must be within distance $k$ to an access of $y$. The reuse distance of the second $x$ access would have been less than or equal to $2 k$, contradictory to the fact that we have removed short-distance reuses. For the same reason, $N_{y}$ should be no more than $N_{x}$; therefore, they are equal ${ }^{1}$. Moreover, for each access to $x$, there is one and only one access to $y$ that is within distance $k$.

Let the total distance of all accesses to $x$ (after removing shortdistance reuses) be $S_{x}$ and the similar total distance for $y$ be $S_{y}$. Then $\left|S_{y}-S_{x}\right|$ should be no more than $k N_{x}$. Hence $\left|\frac{S_{y}}{N_{y}}-\frac{S_{x}}{N_{x}}\right| \leq$ $k$. In words, the average reuse distance of $x$ and $y$ differs by no more than $k$. Although intuitive, the statement needs a non-trivial induction proof, which we do not include for the lack of space. But the conclusion is a pleasing one because the link length $k$ itself bounds the difference of the average distance-the abstract definition and the practical test rely on the same $k$.

When generalized to two sets of data, the necessary condition is slightly different: the average reuse distance of the accesses to the first data set cannot differ more than $k$ from the average distance of the accesses to the second data set. The proof is trivial. The difference of the total distance between the two sets is less than the sum of the difference between each affinity pair, which is $k|N|$, where $|N|$ is total number of accesses to any data set.

The condition is necessary, as shown by the derivation. It is not sufficient. We do not check the reuse distance of each access but only the average distance calculated from the total distance. It is possible that the total distance is the same but individual reuses differ more than $k$ in distance. An improvement is to check the sum of each sub-set of memory accesses instead of the set of all

\footnotetext{
${ }^{1}$ Without going into details, we note that the equality is not strict but holds with arbitrarily high probability determined by $1-\frac{k}{h}$, where $h$ is the threshold for a short distance.
} 
accesses. The difficulty is to partition in the same way for accesses of the two data sets. We use the bins of the reuse signature and check the condition in each bin separately. Considering partition errors at the bin boundaries (an example shown before in Figure 3), we apply the necessary condition to the sum of the average of all bins rather than the average of each bin. The improved condition is as follows.

Let the reuse signature have $B$ bins after removing short-distance bins. Let $X$ and $Y$ be the two sets of data, and $A v g_{i}^{X}$ and $A v g_{i}^{Y}$ be the average reuse distance of the two data sets in the $i$ th bin.

$$
d=\sum_{i=1}^{B}\left|A v g_{i}^{X}-A v g_{i}^{Y}\right| \leq k \cdot B
$$

The equation ensures that the average reuse distance per bin differs no more than $k$. The left-hand side of the inequality is the difference between $X$ and $Y$ known as the Manhattan distance of the two vectors. For two-dimensional vectors, it is the driving distance of a taxi going from one place to another in downtown New York City (no relation to the driving time, however).

In addition, reuse distance does not include the exact time of the data access. It is possible that two elements are accessed in the same reuse distance, but one in the first half of the execution, and the other in the other half. An improvement is to divide the execution trace into sub-parts and check the condition for each part.

When an affinity group has more than two members, the maximal difference between any two members of a $g$-element affinity group is no more than $\frac{g k}{2}$. The condition becomes recursive because knowing group members requires knowing the group size first. The recursive equation can be solved iteratively. For each data set $X$, we find all other sets whose average distance differs no more than $b k$ and let $b$ range from 1 to $\frac{g}{2}$, where $g$ is the number of all data sets. The solution is the largest $b$ such that exactly $b-1$ data sets satisfy the condition. The solution must terminate, and the result correct.

In practice, we use a stricter condition to build a group incrementally. Initially each data set is a group. Then we traverse the groups and merge two groups if a member in one group and another member in the other group satisfy Equation 1. The process terminates if no more groups can be merged. We need to calculate the distance difference between any two data sets in $O\left(g^{2}\right)$ time. The iterative solutions takes at most $O\left(g^{2}\right)$. The incremental solution takes linear time if implemented using a work-list.

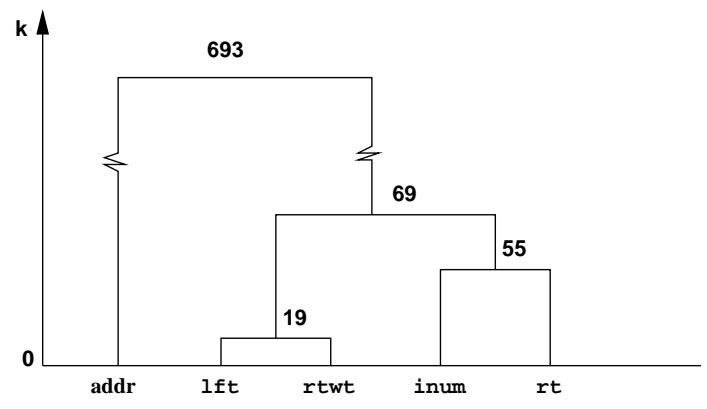

Figure 4: Dendrogram from $k$-distance clustering for the five tree node fields in Cheetah

The reference affinity forms a hierarchy for different $k$ values. Figure 4 shows the hierarchy in a dendrogram. The reference affinity between $l f t$ and $r t w t$ is the strongest- their accesses are within a distance of 19 data elements. Other two fields, inum and $r t$, are used together within a distance of 55. These two groups are reused within $k=69$. The last field, $a d d r$, has least reference affinity, not accessed with other fields within hundreds of data.

\subsection{Comparison with other methods}

The discussion of other methods follows their chronological order and high-lights the three unique features of $k$-distance analysisthe use of reuse signatures, the use of the absolute closeness, and the use of the constant bound $k$. An experimental comparison follows in Section 5.

\subsubsection{Access frequency}

Often in programs some data are more frequently used than others. Clustering the high frequency data would reduce the working set of a program. However, a question remains on how to place data that have the same access frequency, including the (larger) infrequently accessed data. Frequency is not the same as affinity. Data having the same frequency may not be accessed together frequently. They may not be accessed together at all. Reference affinity gives a finer partition and sub-divides data based on the distance of data reuse. It is important to measure distance by volume not time. An execution may nibble small data bits or devour huge data sets in the same amount of time. Reuse signature allows us to remove short-distance reuses as noises, regardless of their frequency or time distance.

An extension to frequency is pair-wise affinity-the frequency that a pair of data are used together. The pair-wise affinity forms a complete graph where each datum is a node and the pair-wise frequency is the edge weight. However, reference affinity is not transitive in this graph. Consider the access sequence $a b a b . . a b \ldots b c b c . . b c$ : the pair-wise affinity exists for $a$ and $b$, for $b$ and $c$, but not for $a$ and $c$. Hence pair-wise affinity is indeed pair wise and cannot tell how to group more than two data elements. Furthermore, the criterion for two data to be "accessed together" is arbitrary. A single "cutoff" radius cannot model affinity relation in a multi-layer hierarchy. In comparison, $k$-distance analysis defines affinity in data groups and measures the "togetherness" using a scale - the data volume between accesses.

\subsubsection{Compiler analysis}

For programs written in regular loops and array access, a compiler can determine the exact access pattern and the best computation and data organization. However, for programs with complex control flow and indirect data access, a compiler must make conservative assumptions to ensure the benefit of a transformation. Profiling analysis is more generally applicable. However, results from one profile may not reflect the access pattern in other executions. $K$-distance analysis alleviates this problem by relying on long reuse distances and the reuse signature, which often have consistent patterns across data inputs. In addition, the analysis considers the access pattern in multiple inputs (by merging reuse signatures) and further reduce the chance of a false positive. The negative conclusion from the analysis, i.e. two data do not have close reference affinity, is always true for the program as a whole: the data violate the necessary condition in some control flow path.

\subsubsection{Statistical clustering}

Since the reuse signature is a vector, a natural impulse is to apply the sophisticated and readily available clustering techniques based on well-grounded multivariate statistical theory. First proposed by MacQueen in 1967, k-means groups high-dimensional vectors by 
minimizing the within-group sum of distances to the group centroid [14]. It iteratively regroups points until a local minimum is reached [10]. It requires $k$, the number of groups, be part of the input. Affinity analysis, however, cannot pre-determine the number of affinity groups. An extension, x-means, finds $k$ automatically [17]. It uses Bayesian Information Criterion (BIC) to compare different groups formed for different $k$ 's and choose the one with the highest probability.

In our preliminary study, k-means and x-means proposed many candidates, a few showed good improvements [24]. However, their results were beyond our comprehension. Little consistency exists between the best grouping of $t$ clusters, $t+1$, and $t-1$ clusters: elements seemed to have regrouped at random. Moreover, for the same number of clusters, k-means produced entirely different groups than $\mathrm{x}$-means did. Part of the problem, as we learned, is that statistical clustering uses relative closeness. The grouping of any two points is determined, ironically, not by their position but by the position of all other points. Figure 5 shows two vector spaces each containing three points. The grouping of the two points, $X$ and $Y$, completely depends on the position of third point $Z$. We can (and we did) alleviate the problem by introducing anchor points into the space, but we also realized that the right measure is the absolute closeness, not the relative closeness. For example, the benefit of grouping two data should be determined how they are accessed, not by other data that may not even be used at all in the same part of the execution. By using absolute closeness, we no longer need these unwieldy statistical tools.

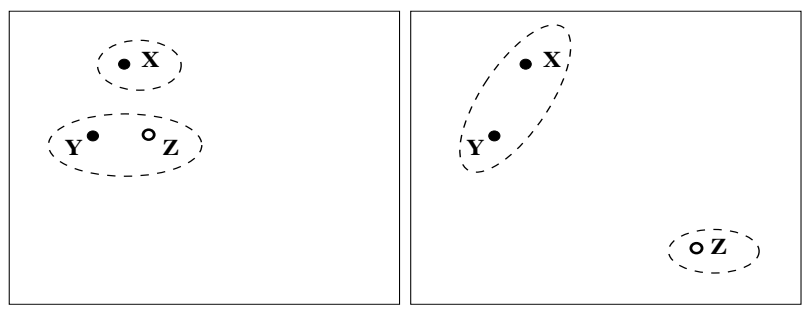

Figure 5: Example of statistical clustering. The grouping of $X$ and $Y$ is determined not by their positions but by the position of $Z$.

\subsubsection{K\%-clustering}

$K \%$-clustering groups two reuse signatures $X$ and $Y$ (of length $B$ ) if the difference $p$, shown below, is less than $k \%$. The difference in each bin, $\left|x_{i}-y_{i}\right|$, can be in the number of reuses, the sum of the reuse distance, or both.

$$
p_{<X, Y>}=\frac{\sum_{i=1}^{B}\left|x_{i}-y_{i}\right|}{\sum_{i=1}^{B} x_{i}+\sum_{i=1}^{B} y_{i}} \times 100 \%
$$

$K \%$-clustering is an improvement over statistical clustering. It measures the absolute closeness between reuse signatures. The result is tangible-the distance difference is no more than $k \%$. The partitions are hierachically nested. If we decrease the value of $k \%$, clusters resulted from a lower $k$ must be a finer partition of those from a higher $k$. The problem, however, is that the test, $k \%$, is relative to the reuse distance. Consider two elements in a $1 \%$ group. The first is always reused by a distance of 100 million. The reuse distance of the second element can range from 99 million to 101 million, therefore having little chance of staying in cache with the first element. The solution is in our definition of reference affinity: the link length should be constant not relative.

We describe $k \%$ clustering as it was in our study-a half-way solution. By comparing $k$-distance and $k \%$-clustering, we show the importance of the constant bound. The bound $k$ plays the most critical role in the affinity definition and the affinity test. This strong link is the most salient feature of this work. It unites the theory and practice of reference affinity.

\section{DATA REORGANIZATION}

Programs often have a large number of homogeneous data objects such as molecules in a simulated container and nodes in a search tree. Each object has a set of attributes. In Fortran 77, attributes of an object are stored separately in arrays. In C, they are stored together in a structure. Neither data layout is sensitive to the access pattern of a program. A better way is to group attributes based on their reference affinity. For arrays, the transformation is array regrouping. For structures, it is structure splitting. This section describes the two transformation and their compiler support.

\subsection{Array regrouping}

Figure 6 shows an example. Initially the three coordinates of $M$ molecules are stored in three arrays $X, Y$, and $Z$. They can be grouped into a single array whose first dimension has 3 elements, equivalent to an array of $M$ structures. Fortran 90 and $\mathrm{C}$ allows grouping arrays of different types by using structures. Array regrouping can be automatically applied in Fortran programs by a compiler [6,7]. We use the same compiler support in this work.

$$
\text { real*8X(M), Y(M), Z(M) real*8XYZ(3,M) }
$$
(a) before grouping
(b) after grouping

Figure 6: Array regrouping example in Fortran 77

\subsection{Structure splitting}

Structure splitting cannot yet be automated for general C programs, so previous work applied it by hand [3,4]. We need to test a number of non-trivial $\mathrm{C}$ programs. To reduce the programming effort and ensure the correctness of the transformed programs, we build a compiler that handles static type-safe $\mathrm{C}$ programs. The compiler support is just enough to evaluate reference affinity in experiments. It is not our purpose in this paper to develop a general technique for structure splitting or to argue that a general solution exists.

In static type-safe $\mathrm{C}$ programs, a compiler knows the correct type of every memory access. Given a program and a structure type targeted for splitting (we call it a split type), the compiler changes the allocation and the access of all objects of the split type (we call them split objects). It first pre-allocates space in field arrays. The allocation of a split object is converted to taking available entries in field arrays. A split object is no longer identified by a pointer but by its array index. Figure 7 shows an example where the three fields of a structure type in Part (a) are split into two groups. Two field arrays are pre-allocated in Part (b) as arrays of structures. The pointers to a split object are changed to integer indices in the new program.

As object pointers become array indices, object access becomes array access (and a structure access if the array contains multiple 


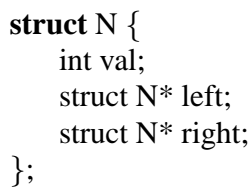

(a) before splitting

\author{
struct $\mathrm{N} \_$fragm0 \{ \\ int val; \\ \}; \\ unsigned int left; \\ struct $\mathrm{N} \_$fragm1 \\ \}; \\ unsigned int right; \\ struct N_fragm0 f0_store[RESERVED]; \\ struct N_fragm1 f1_store[RESERVED];
}

(b) after splitting

Figure 7: Structure splitting example in C

fields). Through type analysis, the compiler knows all the places where a split object is accessed and makes conversion in those and only those places. Taking the pointer of a field in a split object is allowed, which returns a pointer to an element in a field array.

Three problems immediately arise with this scheme. First, a split object can be local to a function and should not permanently stay in field arrays. The compiler solves this problem by managing the upper-end of the field array as a stack and inserting allocation and free calls at the entry and the exit of the function. A field array is then shared by global and stack allocated objects in the same way as virtual memory is shared by heap and stack data, except that we manage a small stack for each split type.

The second problem happens when a split object is nested as a child inside a parent object. Three cases may happen. When the parent is split but the child is not, we support it by not splitting inside a field if it is a structure. When the parent and the child both split, we convert the child field into a pointer, which points to an independent object allocated when the parent object is allocated. We do not support the third case when the child is split but the parent is not.

Another major problem is the size of pre-allocation. The conversion of pointers to indices actually enables a solution through dynamic re-allocation. During execution when the pre-allocated space is full, an allocator doubles each field array by allocating twice the space, copying the old array to the new space, change the base of the field array, and free the old array. The object access is unimpeded after re-allocation. To support array re-allocation, the compiler can no longer let pointers be taken from a field of a split object. In our test programs, pre-allocation is not an obstacle because the programmer specifies a bounded size for the main data structure, as done in all performance-critical programs. For example, Cheetah sets the maximal size of the splay tree by a compile-time constant. None of our programs needs re-allocation at run time. We note that our scheme ignores a host of issues such as union types, long-jump, exceptions, and concurrency because they do not arise in our test programs.

One type of splitting is no splitting, where the compiler does not change the layout of object fields, but it converts split objects from the pointer form to the array form. So far the conversion improves the program speed, often by a double-digit percentage. Just before this submission, however, we found one exception where for one particular input, the array form of Cheetah ran slower than the pointer form. We will investigate further and report the findings in the full paper. In the evaluation, the base time of structure splitting is the time of the array allocated version with no splitting.
Chilimbi et al. are among the first to use structure splitting to improve data locality [4]. For type safe programs, they split an object into two parts, one holding frequently accessed fields and the other holding the rest. A pointer is inserted into the first part to link to the second part. In comparison, array allocation does not support dynamic memory management as transparently as their method does. In the absence of the allocation problem, however, our scheme has better performance because it avoids the space and time cost of the additional pointer. More importantly for this work, array allocation supports arbitrary structure splitting, which is needed for evaluating reference affinity.

\section{EVALUATION}

This section evaluates $k$-distance analysis and compares it with the alternative data layouts given by the programmer and four other methods described in Section 3.3.

\subsection{Methodology}

Test programs. Table 1 lists a set of 9 programs. The first four are array-based programs, including two from Spec95 suite and two dynamic programs originally from the Chaos group [5] for unstructured mesh and N-body simulation. The rest five programs use different tree structures including a splay tree for cache simulation, a quad-tree for image processing, and binary search trees for sorting and various other purposes. The cache simulator is part of the SimpleScalar tool set. The other four programs come from Olden benchmark set. We use different inputs for training and testing, as listed in the table. For a fair comparison, we only use one training input for each benchmark, despite that $k$-distance analysis can use multiple training runs.

We do not test more programs in part because our current compiler cannot not safely split structures in all Olden programs, but also in part because we believe the current set is large and diverse enough. All programs have at least 3 fields or arrays, five have 7 or more, and one program, Swim, has 14 arrays. A program with $n$ arrays or structure fields has an exponential number (at least $2^{n-2}$ and $\sum_{i=0}^{n} i^{(n-i)}$ to be exact) of possible data layouts. The possible layouts is 4 for $n=3,210$ for $n=7$, and over 6 million for $n=14$. Therefore, the probability for a single method to consistently pick the best layout among all choices for all programs is effectively zero, unless the method is indeed the optimal or extremely close to the optimal method.

Platforms. The experiments use two machines, a $1.3 \mathrm{GHz}$ IBM Power4 processor with AIX Compiler, and a $2 \mathrm{GHz}$ Intel Pentium IV processor with Linux $g c c$ compiler. All testing programs are compiled at optimization level -O5 -qstrict with AIX and -O3 with Linux gcc respectively. The time is for complete executions on unloaded processors. We take the shortest time in 5 runs. We also tested programs on a $250 \mathrm{MHz}$ MIPS R10K processor on SGI Origin2000 using the MIPSpro compiler and on a $336 \mathrm{MHz}$ UltraSparc processor using the Sun compiler at the highest optimization level. The qualitative results are similar. The best layout on IBM is also the best on SGI or Sun. The programs run up to five times slower on SGI and many more times slower on Sun. The improvement is less dramatic because the memory problem is less severe on the two slower processors. We do not report SGI and Sun results for the lack of space. 
Table 1: Benchmark characteristics

\begin{tabular}{|c|c|c|c|c|c|}
\hline Benchmark & Source & Description & Main data structure & Training input & Testing input \\
\hline Swim & Spec95 & shallow water equation & 14 real arrays & test $\left(128^{2}\right)$ & ref $\left(512^{2}\right)$ \\
\hline Tomcatv & Spec95 & vectorized mesh generation & 9 real arrays & test $\left(513^{2}\right)$ & $r e f\left(513^{2}\right)$ \\
\hline Mesh & Chaos Group & mesh structure simulation & 7 float arrays & $10 \mathrm{k}$ & $10 \mathrm{k}$ \\
\hline MolDyn & Chaos Group & molecular dynamics simulation & 3 double arrays & 13500 molecules & 62500 molecules \\
\hline \hline Cheetah & SimpleScalar & fully associative LRU cache simulator & splay tree, 5 fields & jpeg encode & jpeg encode \\
& & & & $21.8 \mathrm{~K}$ image & $272 \mathrm{~K}$ image \\
\hline Bisort & Olden & forward \& backward integer sorting & binary tree, 3 fields & $2^{17}$ nodes & $2^{21}$ nodes \\
\hline Perimeter & Olden & perimeters of regions in images & quad-tree, 7 fields & 12 levels & 12 levels \\
\hline TreeAdd & Olden & recursive sum of values in a balanced-tree & binary tree, 3 fields & $2^{18}$ nodes & $2^{22}$ nodes \\
\hline TSP & Olden & traveling salesman problem solver & binary tree, 7 fields & $10^{5}$ nodes & $10^{6}$ nodes \\
\hline
\end{tabular}

Tools. We use source-level instrumentation and a run-time monitor to profile accesses for individual arrays and structure fields [7, 8]. It is more accurate and efficient than other tools because it finds the exact logical data element being accessed without searching through all run-time data objects. We use a 99\%-accurate analyzer for reuse distance analysis [9]. It measures reuse distance in arbitrarily long traces in almost linear time and guarantees that the measured distance is between $99 \%$ and $100 \%$ of the actual distance. These two results then give the reuse signature for each array or structure field. We implement $k$-distance, $k \%$, and frequencybased analysis in MATLAB. The k-means and $\mathrm{x}$-means tools come from Pelleg and Moore [17]. Compiler-based array regrouping is the same as the one given by Ding and Kennedy [7]. We implement array regrouping and structure splitting as described in Section 4.

\subsection{Performance Comparison}

We compare eight data-layout methods. The results are shown in Table 2 for the PC and Table 3 for the IBM machine. All execution times are in seconds. The last row is the arithmetic mean of the speedup. The first is the original layout coded by the programmer. The next two are $k$-distance for $k=256$ and 64 , followed by two $k \%$ methods for $1 \%$ and $0.1 \%$. Then is $x$-means. We do not include $k$-means because it cannot determine the number of affinity groups. The seventh method uses access frequency to divide data into groups. We cluster the smallest set that accounts for more than $50 \%$ of all accesses into one group and leave the others in singleelement groups. The last method uses the compiler analysis given by Ding and Kennedy [7]. The same data layout is evaluated on both machines for each method.

The $k$-distance method for $k=256$ should be the best method. It groups data that are almost always used within one to two kilobytes of data access, which fits comfortably in the L1 cache of all machines we use. For large cache, $k$ should be greater. We stick to a single value because all other methods use a single parameter. Indeed, the analysis picks the best data layout for all programs on the two machines. Swim has 14 arrays and over 6 million possible choices, the analysis singles out a layout, which outperforms all others by a wide margin. The affinity hierarchy of Swim is a very impressive, large tree, as shown by Figure 8 . On the PC, the layout from $k$-distance is $4 \%$ faster than $x$-means, $19 \%$ than compiler analysis, $20 \%$ or more than others, and $37 \%$ faster than the original layout. The improvement is smaller on IBM, $14 \%$ faster than the original. However, the margin is indisputable-no other known layout comes within $91 \%$ performance of this seemingly singular choice by the analysis.

For other programs, 256-distance improves Tomcatv by $27 \%$,

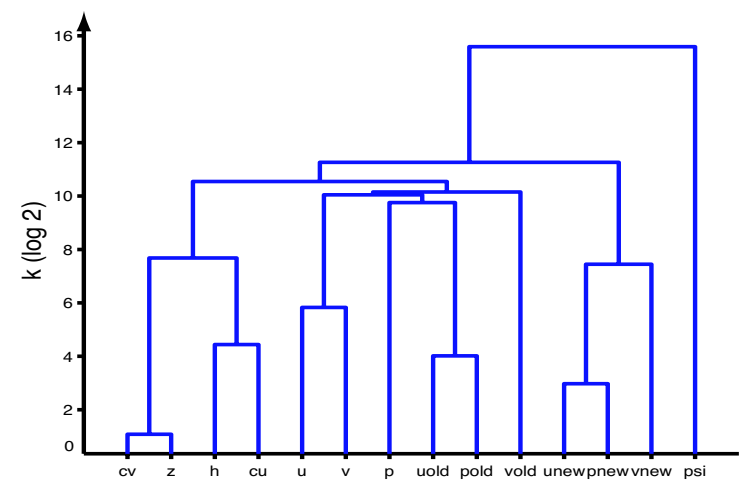

Figure 8: Dendrogram from $k$-distance for Swim

TSP by $18 \%$, and Cheetah by $9 \%$ on the PC. The average improvement is $12 \%$ on the PC and $6 \%$ on IBM. The improvement is more significant for programs that have many arrays or fields. The analysis does not blindly transform a program. The arrays in MolDyn and the structure in Bisort given by the programmer have the best performance. The analysis recommends no change.

256-distance analysis runs slower in only three places, TreeAdd against the original on the PC, Tomcatv against $\mathrm{x}$-means on IBM, and Perimeter against 64-distance on the PC. However, the loss is extremely small (no more than $1.5 \%$ or 0.004 seconds), happens on only one machine, and is more than compensated by the improvement on the other machine if we take the average. For TreeAdd against the original, it loses by $0.7 \%$ on the PC but wins by $13 \%$ on IBM. Except for them, 256-distance always picks the best data layout when competing against all 7 other methods for all 9 programs and 2 machines. It ties or wins in $98 \%$ of all contests. When measured by the average speed on the two machines, it never loses.

The layout by $k$-distance with $k=64$ is overly conservative because it does not exploit affinity at a distance greater than 64 . It picks the same data layout as $k=256$ for all but three programs, suggesting that the affinity groups have a short distance in majority of the programs.

The two $k \%$-clustering methods give competitive results, improving average performance by $7 \%$ on the PC and $5 \%$ on IBM. The two $k$ values, $k=1 \%$ and $k=0.1 \%$, give the same layout in all but three programs, of which $k=1 \%$ wins in two but loses in one, on both machines. This verifies that no single $k \%$ is the best, because $k \%$ does not have absolute meaning and may be too small in one program but too large in another. In contrast, the $k$ in 
Table 2: Execution time (sec) on Intel Pentium IV

\begin{tabular}{|c|c|c|c|c|c|c|c|c|}
\hline Benchmark & Orig & $K=256$ & $K=64$ & $K=1 \%$ & $K=0.1 \%$ & X-means & Freq $=50 \%$ & Static \\
\hline Swim & 52.84 & 38.52 & 53.65 & 47.59 & 53.65 & 40.07 & 46.32 & 45.9 \\
\hline Tomcatv & 46.13 & 36.43 & 36.43 & 36.43 & 36.43 & 37.65 & 37.35 & 38.03 \\
\hline MolDyn & 69.78 & 69.78 & 69.78 & 69.78 & 69.78 & 69.79 & 69.78 & 69.78 \\
\hline Mesh & 4.31 & 4.27 & 4.31 & 4.27 & 4.31 & 5.29 & 5.71 & 4.31 \\
\hline Cheetah & 20.67 & 18.91 & 18.91 & 18.91 & 18.91 & 20.85 & 20.54 & \multirow{6}{*}{$\begin{array}{c}\text { compiler } \\
\text { analysis } \\
\text { not } \\
\text { applicable }\end{array}$} \\
\hline Bisort & 13.23 & 13.23 & 13.23 & 13.23 & 13.23 & 14.38 & 14.38 & \\
\hline Perimeter & 0.035 & 0.030 & 0.026 & 0.030 & 0.030 & 0.030 & 0.030 & \\
\hline TreeAdd & 0.262 & 0.264 & 0.264 & 0.264 & 0.264 & 0.264 & 0.264 & \\
\hline TSP & 4.37 & 3.71 & 3.71 & 4.18 & 3.74 & 4.17 & 3.71 & \\
\hline Average Speedup & 1.000 & 1.120 & 1.096 & 1.076 & 1.075 & 1.053 & 1.044 & \\
\hline
\end{tabular}

Table 3: Execution time (sec) on IBM Power 4

\begin{tabular}{|c|c|c|c|c|c|c|c|c|}
\hline Benchmark & Orig & $K=256$ & $K=64$ & $K=1 \%$ & $K=0.1 \%$ & X-means & Freq $=50 \%$ & Static \\
\hline Swim & 27.24 & 24.00 & 26.08 & 27.88 & 26.08 & 26.79 & 27.44 & 27.32 \\
\hline Tomcatv & 21.74 & 20.92 & 20.92 & 20.92 & 20.92 & 20.61 & 23.79 & 21.02 \\
\hline MolDyn & 52.22 & 52.22 & 52.22 & 52.22 & 52.22 & 52.29 & 52.22 & 52.22 \\
\hline Mesh & 3.29 & 3.26 & 3.29 & 3.26 & 3.29 & 3.32 & 3.42 & 3.29 \\
\hline Cheetah & 18.23 & 17.02 & 17.02 & 17.02 & 17.02 & 17.94 & 17.15 & \multirow{6}{*}{$\begin{array}{l}\text { compiler } \\
\text { analysis } \\
\text { not } \\
\text { applicable }\end{array}$} \\
\hline Bisort & 8.07 & 8.07 & 8.07 & 8.07 & 8.07 & 10.71 & 10.26 & \\
\hline Perimeter & 0.025 & 0.022 & 0.027 & 0.022 & 0.022 & 0.022 & 0.024 & \\
\hline TreeAdd & 0.256 & 0.226 & 0.226 & 0.226 & 0.226 & 0.226 & 0.226 & \\
\hline Tsp & 10.04 & 9.80 & 9.80 & 9.83 & 9.81 & 9.95 & 9.80 & \\
\hline Average Speedup & 1.000 & 1.061 & 1.026 & 1.043 & 1.050 & 1.012 & 0.991 & \\
\hline
\end{tabular}

$k$-distance has an absolute meaning - the reuse distance.

$\mathrm{X}$-means, using relative closeness, performs worse than all methods using absolute closeness. The average improvement is $5 \%$ on the PC and $1 \%$ on IBM. Its layout choice has bewilderingly different quality on two machines. It gives the second best data layout for Swim on the PC, but the same layout is the second slowest on IBM. The three fields of Bisort have almost identical reuse signatures. Based on the relative closeness, $\mathrm{x}$-means stubbornly splits them, losing performance by $9 \%$ on the PC and $33 \%$ on IBM.

Frequency splitting using the single parameter performs worse on average than all other methods, showing that grouping on frequency is not as good as grouping on reuse signatures. Compiler analysis is conservative. It causes no slowdown but has much less benefit than $k$-distance analysis. It cannot yet analyze structure access in $\mathrm{C}$ programs.

An interesting use of $k$-distance analysis is to find the worst data layout by reversing the reference affinity. It has no practical use other than showing the range of the effect from the data layout. We are still collecting data in some part because the experiment takes too long. We will report the findings in the full paper.

Miss-rate improvement across all inputs. The effect of data transformation may change with program inputs. Our past work showed that Tomcatv has a predictable miss rate across all inputs [23]. Using that tool, we draw the miss rate of $96 \mathrm{~KB}$ cache for the original and $k$-distance analysis with $k=256$ as two curves in Figure 9. The data input is measured by the data size in cache blocks. Array regrouping reduces miss rate by $0 \%$ to over $5 \%$ depending on the input. The vertical bar gives the data input used in our experiments. The difference is about $0.7 \%$ in absolute miss rate.

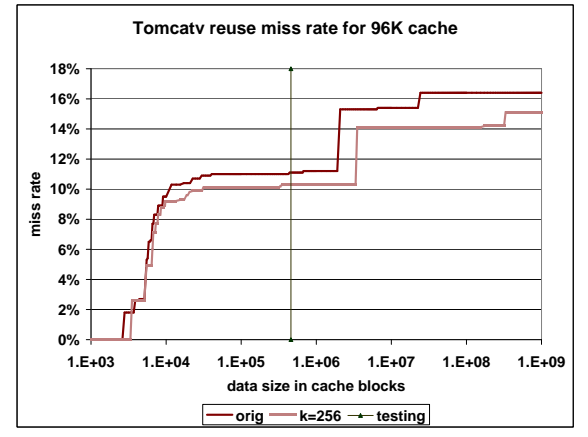

Figure 9: Reuse miss rate changes for Tomcatv

\section{RELATED WORK}

This section discusses in two groups only past work on data transformation: one based on reference affinity using mainly compiler analysis, and the other on access frequency using profiling. The discussion is more bibliographical than technical. The reader should refer Section 3.3 for a technical comparison.

Early compiler analysis identifies groups of data that are used together in loop nests, using the concepts of reference approximity [21] and reference groups [16, 22]. Thabit showed that optimal pair-wise affinity placement is NP-hard [21]. Kennedy and Kremer gave a general model that considered, among others, run-time data transformation. They showed that the problem is also NP-hard [12]. Ding and Kennedy used their results to prove the complexity of partial and dynamic reference affinity [6]. To reduce false sharing in 
multi-treaded programs, Anderson et al. [1] and Eggers and Jeremiassen [11] grouped data accessed by the same thread. Anderson et al. optimized for computation as well as data locality, but they did not combine different arrays. Eggers and Jeremiassen combined multiple arrays for thread locality, but their scheme may hurt cache locality if not all thread data are used at the same time.

For improving cache performance, Ding and Kennedy grouped arrays that are always used together in a program [6]. They gave the optimal array layout for strict affinity. They later grouped arrays at multiple granularity [7]. An earlier version of this work defined hierarchical reference affinity and tested two programs using $\mathrm{x}$-means and k-means clustering [24].

Program profiling has long been used to find the frequency of data access [13]. Seidl and Zorn grouped frequently accessed objects to improve virtual memory performance [19]. Calder et al. modeled pair-wise affinity using temporal relation graphs and developed an algorithm for placing data variables and objects [2]. The two methods did not recombine data across object boundaries. Chilimbi et al. are among the first to use structure splitting to improve data locality [4]. Chilimbi later used the frequency of data sub-streams called hot-streams [3]. Hot-streams combines dynamic affinity with frequency but does not yet give whole-program reference affinity.

Access frequency and pair-wise affinity do not distinguish the time or distance of reuse. Petrank and Rawitz formalized this observation and proved a harsh bound: with only frequency or pairwise information, no algorithm can find a static data layout that is always within a factor of $k-3$ from the optimal solution, where $k$ is proportional to the size of cache [18].

\section{CONCLUSIONS}

In this paper we have defined reference affinity, which gives a unique and hierarchical partition of all data. We have presented $k$ distance for program affinity analysis and compared it with other techniques including manual layout, frequency profiling, compiler analysis, and statistical clustering. The new method has rigorous properties owing to its direct link, $k$, to the affinity definition. Experiments have shown that the new method reveals rich affinity relationship among program data, which forms a hierarchy of detailed layers with a different but intuitive distance $k$. In array and structure reorganization, $k$-distance analysis outperforms all other methods with remarkable consistency. The close agreement between theoretical properties and experimental observations suggests that reference affinity is an effective way to bridge the gap between the memory hierarchy of a machine and the linear data layout of a program.

\section{ACKNOWLEDGMENTS}

This work is supported by the National Science Foundation (Contract No. CCR-0238176, CCR-0219848, and EIA-0080124) and the Department of Energy (Contract No. DE-FG02-02ER25525).

\section{REFERENCES}

[1] J. Anderson, S. Amarasinghe, and M. Lam. Data and computation transformation for multiprocessors. In Proceedings of the Fifth ACM SIGPLAN Symposium on Principles and Practice of Parallel Programming, Santa Barbara, CA, July 1995.

[2] B. Calder, C. Krintz, S. John, and T. Austin. Cache-conscious data placement. In Proceedings of the Eighth International Conference on Architectural Support for Programming Languages and Operating Systems (ASPLOS-VIII), San Jose, Oct 1998.
[3] T. M. Chilimbi. Effi cient representations and abstractions for quantifying and exploiting data reference locality. In Proceedings of ACM SIGPLAN Conference on Programming Language Design and Implementation, Snowbird, Utah, June 2001.

[4] T. M. Chilimbi, B. Davidson, and J. R. Larus. Cache-conscious structure defi nition. In Proceedings of ACM SIGPLAN Conference on Programming Language Design and Implementation, Atlanta, Georgia, May 1999.

[5] R. Das, M. Uysal, J. Saltz, and Y.-S. Hwang. Communication optimizations for irregular scientifi c computations on distributed memory architectures. Journal of Parallel and Distributed Computing, 22(3):462-479, Sept. 1994.

[6] C. Ding and K. Kennedy. Inter-array data regrouping. In Proceedings of The 12th International Workshop on Languages and Compilers for Parallel Computing, La Jolla, California, August 1999.

[7] C. Ding and K. Kennedy. Improving effective bandwidth through compiler enhancement of global cache reuse. In Proceedings of International Parallel and Distributed Processing Symposium, San Francisco, CA, 2001. http://www.ipdps.org.

[8] C. Ding and Y. Zhong. Compiler-directed run-time monitoring of program data access. In Proceedings of the fi rst ACM SIGPLAN Workshop on Memory System Performance, Berlin, Germany, June 2002.

[9] C. Ding and Y. Zhong. Predicting whole-program locality with reuse distance analysis. In Proceedings of ACM SIGPLAN Conference on Programming Language Design and Implementation, San Diego, CA, June 2003.

[10] J. A. Hartigan. Clustering Algorithms. John Wiley \& Sons, 1975.

[11] T. E. Jeremiassen and S. J. Eggers. Reducing false sharing on shared memory multiprocessors through compile time data transformations. In Proceedings of the Fifth ACM SIGPLAN Symposium on Principles and Practice of Parallel Programming, pages 179-188, Santa Barbara, CA, July 1995.

[12] K. Kennedy and U. Kremer. Automatic data layout for distributed memory machines. ACM Transactions on Programming Languages and Systems, 20(4), 1998.

[13] D. Knuth. An empirical study of FORTRAN programs. Software-Practice and Experience, 1:105-133, 1971.

[14] J. MacQueen. Some methods for classifi cation and analysis of multivariate observations. In Proceedings of 5th Berkeley Symposium on Mathematical Statisitics and Probability, pages 281-297, 1967.

[15] R. L. Mattson, J. Gecsei, D. Slutz, and I. L. Traiger. Evaluation techniques for storage hierarchies. IBM System Journal, 9(2):78-117, 1970.

[16] K. S. McKinley, S. Carr, and C.-W. Tseng. Improving data locality with loop transformations. ACM Transactions on Programming Languages and Systems, 18(4):424-453, July 1996.

[17] D. Pelleg and A. Moore. X-means: Extending k-means with effi cient estimaiton of the number of clusters. In Proceddings of the 17th International Conference on Machine Learning, pages 727-734, San Francisco, CA, 2000.

[18] E. Petrank and D. Rawitz. The hardness of cache conscious data placement. In Proceedings of ACM Symposium on Principles of Programming Languages, Portland, Oregon, January 2002.

[19] M. L. Seidl and B. G. Zorn. Segregating heap objects by reference behavior and lifetime. In Proceedings of the Eighth International Conference on Architectural Support for Programming Languages and Operating Systems (ASPLOS-VIII), San Jose, Oct 1998.

[20] X. Shen, Y. Zhong, and C. Ding. Regression-based multi-model prediction of data reuse signature. In Proceedings of the 4th Annual Symposium of the Las Alamos Computer Science Institute, Sante Fe, New Mexico, November 2003.

[21] K. O. Thabit. Cache Management by the Compiler. PhD thesis, Dept. of Computer Science, Rice University, 1981.

[22] M. E. Wolf and M. Lam. A data locality optimizing algorithm. In Proceedings of the SIGPLAN '91 Conference on Programming Language Design and Implementation, Toronto, Canada, June 1991.

[23] Y. Zhong, S. G. Dropsho, and C. Ding. Miss rate preidiction across all program inputs. In Proceedings of the 12th International 
Conference on Parallel Architectures and Compilation Techniques, New Orleans, Louisiana, September 2003.

[24] Y. Zhong, X. Shen, and C. Ding. A hierarchical model of reference affi nity. In Proceedings of the 16th International Workshop on

Languages and Compilers for Parallel Computing, College Station, Texas, October 2003. 